\title{
Análisis comparativo de dos operadores turísticos en el ACR Albufera de Medio Mundo, Lima - Perú
}

\author{
Comparative analyze of two touristic operators at the \\ ACR Albufera de Medio Mundo, Lima - Peru
}

Jimena E. Prado ${ }^{1, \star}$ y Jorge M. Chávez ${ }^{1}$

Recibido: 23 marzo 2019 | Aceptado: 03 noviembre 2019| Publicado en línea: 15 diciembre 2019 Citación: Prado, JE; Chávez, JM. 2019. Análisis comparativo de dos operadores turísticos en el ACR Albufera de Medio Mundo, Lima - Perú. Revista Forestal del Perú 34(2): 172-190. DOI: http://dx.doi.org/10.21704/rfp.v34i2.1328

\begin{abstract}
Resumen
El uso turístico de calidad ayuda a que los visitantes comprendan los valores intrínsecos del lugar visitado y la necesidad de conservarlo, buscando que desarrollen una actitud favorable hacia la preservación del área. La presente investigación se realizó en el Área de Conservación Regional Albufera de Medio Mundo (ACRAMM), ubicada en el distrito de Végueta, provincia de Huaura, departamento de Lima, Perú. En esta área existen dos alternativas de operadores turísticos que ofrecen servicios similares: hospedaje, alimentación, recorrido turístico y guiado, entre otros. El objetivo del trabajo fue comparar las operaciones y servicios turísticos del operador público versus los del privado, para determinar cuál ofrece una experiencia de visita más satisfactoria en el ACRAMM, con el añadido de ayudar al desarrollo de mayor conciencia de conservación del área. La metodología de evaluación en campo constó de tres componentes: (1) recorridos por el área y entrevistas personales, (2) el diseño y aplicación de una encuesta a los visitantes que escogieron tanto el operador público como el privado para evaluar el grado de satisfacción de la experiencia y su opinión sobre las facilidades, y (3) el diseño y aplicación de una tabla de puntaje denominada "Ficha de Evaluación del Uso Turístico" para que los autores evalúen la calidad de las operaciones turísticas pública y privada. A través de los resultados de los métodos de evaluación, se determinó que el operador turístico privado ofrece servicios turísticos de mayor calidad que el público; es decir que los visitantes reciben una experiencia de visita más satisfactoria; sobre todo porque en el servicio de recorrido turístico y guiado se prioriza la trasmisión de mensajes sobre la importancia de la conservación del sitio.
\end{abstract}

Palabras clave: humedal costero, área natural protegida, satisfacción de la experiencia del visitante, calidad, turismo ecológico, conservación de la naturaleza

\footnotetext{
${ }^{1}$ Departamento de Manejo Forestal, Facultad de Ciencias Forestales, Universidad Nacional Agraria La Molina (UNALM), Av. La Molina s/n, La Molina, Lima, Perú.

* Autor de Correspondencia: jimena prado216@hotmail.com
} 


\begin{abstract}
A better touristic use quality helps the visitors to understand the intrinsic values of the visited place and the necessity of conserve it, looking for them to develop a positive attitude toward the preservation of the area. The current investigation took place at the Regional Conservation Area Albufera de Medio Mundo (ACRAMM), located at the Végueta district, Huaura province, Lima department, Peru. In this protected area, there exist two alternatives of touristic operators that offer similar services: hosting, feeding, tourist and guided tour, among others. The main objective of this research was to compare the touristic operations and services of the public operator versus the private in order to determinate which one offer a more satisfactory visit experience at the ACRAMM, with the additional of helping to develop a greater consciousness of conserve the place. The evaluation methodology on field had three principal components: (1) personal interviews and tours around the area, (2) the design and application of a survey for the visitors that chose both, the public and the private operator, in order to evaluate the degree of satisfaction on the experience visiting the same site and their opinion about the facilities, and (3) the design and application of a scorecard named "Touristic Use Evaluation Sheet" for the authors to evaluate the quality of the public and private's touristic operations. Through the results of the evaluation methods, it was determined that the private operator offer touristic services of higher quality than the public, which means that the visitors have a more satisfactory visit experience, above all because of the guided tour service that prioritizes the transmission of messages about the importance of site conservation.
\end{abstract}

Key words: coastal wetland, natural protected area, satisfaction of the visitor experience, quality, ecotourism; nature conservation

\section{Introducción}

Los humedales distribuidos a lo largo de la costa peruana constituyen una importante reserva de agua dulce en zonas áridas y albergan alta diversidad a nivel de especies y material genético, incluyendo muchas veces especies amenazadas o en peligro de extinción (Moschella 2012). Estos forman, junto con las lomas y los tilandsiales, un corredor de diversidad biológica en el desierto sudamericano (Aponte et al. 2012). Además, siendo la costa una región altamente poblada, las poblaciones humanas se han encontrado con los humedales haciendo uso de sus múltiples bienes y servicios ecosistémicos (Aponte y Cano 2018). Por ejemplo, el uso de recursos naturales aprovechables como la totora y el uso turístico de estos espacios, debido a su gran valor paisajístico. Este tipo de humedales es considerado sumamente importante para el desarrollo de diversas actividades para el hombre, ya que cumplen una serie de funciones que permiten el normal desenvolvimiento del paisaje, como: reserva de agua dulce, acumulación de biomasa y formación de suelos orgánicos, zona de re- carga, almacenamiento y/o de descarga de las aguas superficiales y subterráneas; sostén para la pesca, la ganadería y la agricultura; espacio para actividades educativas y recreativas al aire libre para la sociedad humana; hábitat para fauna y flora, en especial para las aves acuáticas; control de la sedimentación y erosión e incremento de la calidad paisajística y con ello, la posibilidad de oferta turística (ProNaturaleza 2010).

El humedal del Área de Conservación Regional Albufera de Medio Mundo (ACRAMM) desempeña una función de conectividad como Corredor Biológico de la Costa Central de Lima, lo cual le permite reunir una biodiversidad representativa de dicha zona. Asimismo, este ecosistema contribuye a la sostenibilidad de los procesos biológicos y ecológicos y constituye un sitio de importancia para poblaciones de aves migratorias (GORE Lima 2014). También, ha sido identificado como un Humedal de importancia a nivel nacional, incluido en la Estrategia Nacional para la Conservación de Humedales en el Perú (MINAM 2015) e integra el corredor de humedales de importan- 
Análisis comparativo de dos operadores turísticos en el

cia para la conservación de aves migratorias, denominado Corredor Biológico del Pacífico (Decreto supremo $\mathrm{N}^{\circ}$ 006-2007-AG 2007). Por otro lado, posee gran potencial turístico y recreativo en el norte chico de Lima debido a su belleza paisajística y diversidad biológica (Aponte 2017).

El turismo es el uso social que actualmente reclama cada vez mayor atención ya que, al aumentar el volumen de visitas a un atractivo turístico, este puede tener impactos negativos a veces con consecuencias graves, costosas de revertir (CTN PERÚ 2007). También, el turismo es un fenómeno dinámico y en crecimiento que, además de la derrama económica, genera beneficios sociales y culturales al permitir la interrelación entre los visitantes y la comunidad local (Sosa y Silvestre 2018). Por ello, es necesario evaluar las condiciones de visita y servicios ofrecidos a través de variables claras e indicadores cuantificables. La calidad turística se hace indispensable en las actividades empresariales y se refiere al servicio, donde la calidad se mide desde la apreciación del turista, es decir, todo se resumen en la satisfacción y superación de las necesidades y requerimientos que tenga el turista (Castilla y Alarcón 2017). Por tal motivo, el reporte de la experiencia es un dato fundamental en la planeación y evaluación turística (Hernández et al. 2015). Entendiéndose a la experiencia turística como un conjunto de impresiones físicas, emocionales, sensoriales, espirituales y/o intelectuales, que son percibidas de manera diferente por los turistas, desde el mismo momento en que planifican su viaje, lo disfrutan en el destino elegido e incluso cuando vuelven a su lugar de origen y recuerdan su viaje (Otto y Ritchie, citado por Rivera 2013). En este sentido, el ecoturismo puede y debe ser una herramienta para la conservación (Pacífico Adventures 2016); por lo tanto, su desarrollo en áreas naturales protegidas, requiere fundamentalmente de planificación turística para lograr el objetivo principal de conservación y los objetivos secundarios: calidad de servicio, satisfacción de la experiencia del visitante, puesta en valor de los recursos, desarrollo social local y rentabilidad económica. Se recalca la necesidad de nuevas formas de investigación aplicadas al estudio de la experiencia turística, especialmente desde un enfoque cuantitativo, donde se encuentran limitaciones importantes para comprender en profundidad su naturaleza y valoración (Aníbal y Baez 2018).

El 10 de noviembre de 1983, la Albufera de Medio Mundo es declarada como Zona de Reserva Turística mediante la Resolución Suprema No 237-83-ITI/TUR. Trece años más tarde, el 12 de marzo de 1996 se aprueba la "Estrategia Nacional para la Conservación de Humedales en el Perú" mediante la Resolución Jefatural 054-96-INRENA, en la que se incluye a las Lagunas de Medio Mundo en la lista de los principales humedales (MINAM 2015). El 25 de enero del 2007 se establece, mediante el D.S. No 006-2007-AG, el Área de Conservación Regional Albufera de Medio Mundo por ser reconocida por la comunidad científica debido a la importancia que tiene al cumplir funciones específicas para el mantenimiento de los procesos ecológicos a escala hemisférica en lo concerniente a la protección de especies de flora y fauna (Decreto supremo $\mathrm{N}^{\circ} 006-2007-\mathrm{AG}$ 2007), como parte de las áreas complementarias establecidas por el SINANPE. Luego, el 15 de julio del 2010 se aprueba el Plan Maestro 2009-2013 del ACRAMM a través de la Ordenanza Regional No 004-20120-CR-RL, el cual fue actualizado el 30 de diciembre del 2014 como Plan Maestro 2015-2019, mediante la Ordenanza Regional No 09-2014-CR-RL.

El área es administrada e íntegramente financiada por el Gobierno Regional de Lima (Decreto supremo No 006-2007-AG 2007). Sin embargo, quien presta servicios turísticos de hospedaje, recorrido turístico y guiado, información y servicio de alimentación en las instalaciones turísticas construidas por MINCETUR años atrás (GORE Lima 2014) es un operador turístico que se denominará como "operador público".

Además, en la misma área existe otra alternativa turística, con servicios similares, que se denominará se como "operador privado". Hace cincuenta años, el área e instalaciones de esta empresa fueron propiedad del Viceministerio de Pesquería, parte del Ministerio de 
la Producción (PRODUCE), luego fue otorgado a la Empresa Nacional de Edificaciones (ENACE), que entró en liquidación en el año 2006, rematando entre sus activos, 65 ha de la zona norte del ACRAMM, que hace aproximadamente 10 años, pertenecen a la empresa propietaria de dicho complejo hotelero.

Las principales actividades económicas realizadas en el ACRAMM por la población local son: la pesca artesanal, el cultivo y extracción de junco (Schoenoplectus americanus (Pers.) Volkart ex Schinz \& R. Keller) y la totora balsa (Schoenoplectus californicus (C. A. Mey.) Soják) y el turismo, que representa la actividad de mayor potencial en el ACRAMM, debido a su belleza paisajística y diversidad de flora y fauna. Asimismo, el área recibe una creciente cantidad de visitantes nacionales y extranjeros, sobre todo en la temporada de verano y en especial en dos fechas: Año Nuevo y Semana Santa (500-1000 personas) (Procomhcc, citado por GORE Lima 2009).

Por otro lado, una de las principales actividades en el entorno próximo al ACR, es la avicultura a gran escala desarrollada por la empresa Redondos S.A., donde se estima la presencia de aproximadamente 10000 aves por galpón al año, distribuidos a lo largo de la zona este del ACR (GORE Lima 2009). Esto representa una importante amenaza respecto a la sanidad de la laguna, que recibe infiltraciones de agua proveniente de la granja de dicha empresa, lo que, a su vez, significa un riesgo a la salud pública de los visitantes, las aves migratorias y las aves residentes, por estar expuestos a enfermedades como la gripe aviar. Cabe mencionar que, en el Plan Maestro 2015-2019 se ha identificado y analizado, bajo la Matriz de Vester, los principales problemas que influyen en el deterioro ambiental y la calidad de vida de los habitantes, siendo el problema categorizado como "crítico" la baja conciencia ambiental, ya que causa la mayoría de los demás. Asimismo, se menciona que, los actores involucrados directos e indirectos, tanto del sector público como privado, muestran poco interés en realizar actividades de conservación y/o acondicionar sus actividades económicas y cotidianas de mane- ra sostenible con el ambiente. Indicadores de dicha baja conciencia ambiental son: la acumulación de residuos sólidos y desmonte en las inmediaciones, la ausencia de áreas verdes en el centro poblado, inadecuada disposición final de residuos sólidos por los usuarios del área, el vertimiento de efluentes por empresas privadas, la quema de restos vegetales y la sobre pesca de recursos hidrobiológico, entre otros (GORE Lima 2014). La situación actual de riesgo en la que se encuentra el ACRAMM debido a la presión antrópica y el manejo inadecuado de recursos en zonas adyacentes, evidencia la inminentemente necesidad de concientizar a los visitantes sobre la importancia de su conservación para lograr la perpetuidad del espacio a lo largo del tiempo.

Se espera que la metodológica aplicada en esta investigación sirva como una alternativa para evaluar la calidad de los servicios turísticos que ofrecen los operadores turísticos y determinar el grado de satisfacción de los visitantes, de forma que se pueda identificar los aspectos positivos y negativos que contribuyen más o menos al mantenimiento y conservación del ANP relacionada, y así mejorar la calidad de estos. Por ende, el objetivo de esta investigación es comparar las operaciones y servicios turísticos del operador público y el privado en el ACRAMM para determinar cuál ofrece una experiencia de visita de mayor calidad en pro del desarrollo de conciencia sobre la conservación del área.

\section{Materiales y Métodos}

\section{Área de estudio}

La presente investigación se llevó a cabo en el Área de Conservación Regional Albufera de Medio Mundo (ACRAMM), que se encuentra ubicado en el distrito de Végueta, provincia de Huaura, departamento de Lima, Perú. Esta ACR se encuentra a 12 m.s.n.m., a la altura del kilómetro 177 de la carretera Panamericana Norte. El área comprende una extensión de 687,71 ha; de las cuales 206,10 ha contienen al espejo de agua y 66,50 ha la parte pantanosa. El largo es de $5 \mathrm{~km} 775 \mathrm{~m}$ con un ancho que varía 


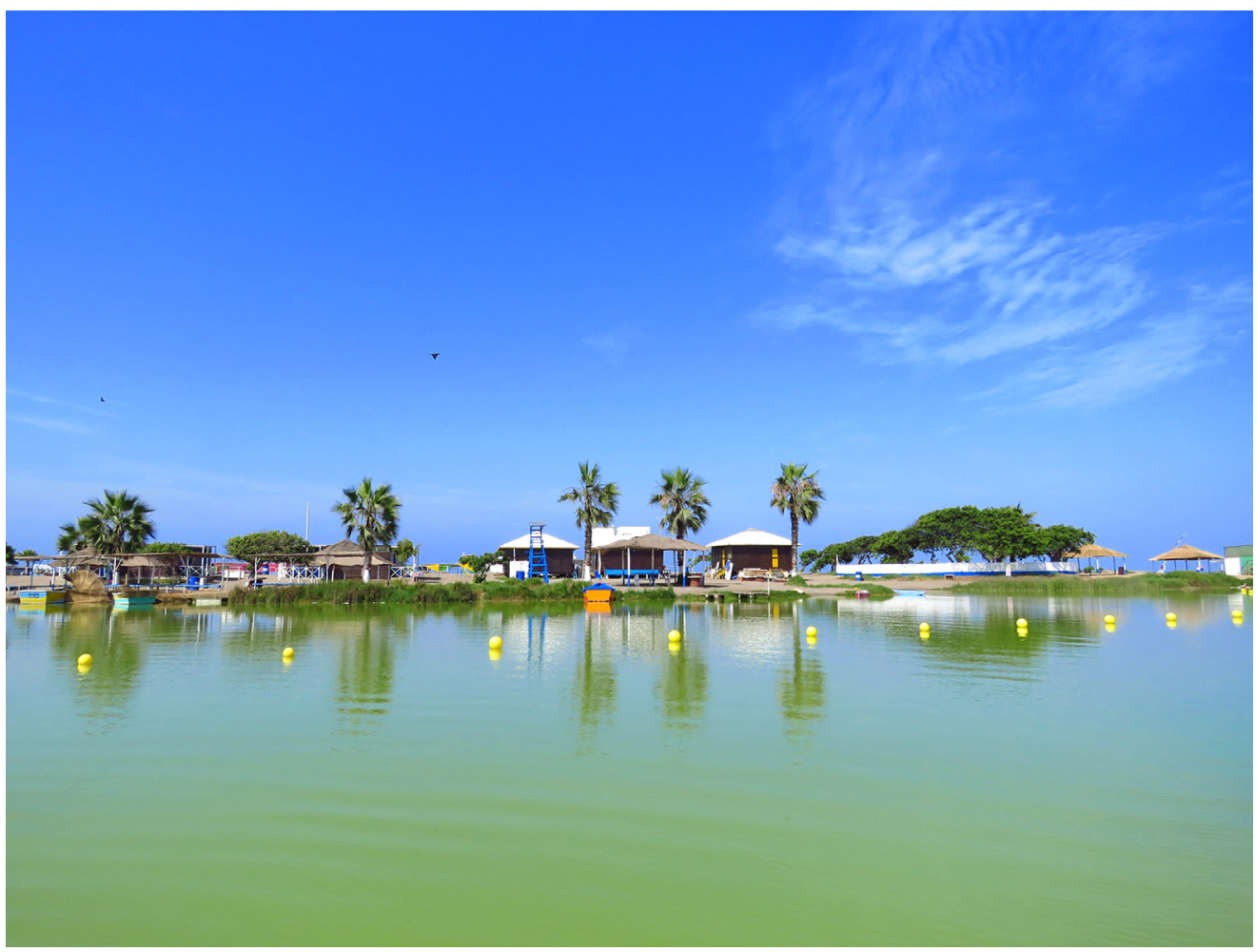

Figura 1. Área de Conservación Regional Albufera de Medio Mundo. Fotografía de Luis Daniel Rojas Quevedo (2018).

de 165 a 525 m (Tovar 1977). Los límites del área son: por el norte con la Playa Los Viños, por el sur con El Centro Poblado Santa Cruz, por el este con la granja de pollos de la empresa Redondos S.A. y el Centro Poblado Medio Mundo, al margen de la carretera Panamericana norte, y por el oeste con el litoral costero.

El acceso al ACR Albufera de Medio Mundo es desde el km 177 de la Panamericana Norte (GORE Lima 2009). Desde ese punto se toma la carretera afirmada que lleva primero, a aproximadamente $1,5 \mathrm{~km}$, a la entrada de las instalaciones del operador privado y luego, a aproximadamente $2,5 \mathrm{~km}$ las instalaciones turísticas del operador público (ver Figura 2).

\section{Toma de datos}

Se evaluó la calidad de los servicios turísticos del operador público y el privado a través de: (1) entrevistas personales dirigidas a personas involucradas directamente con la administración de ambos operadores, (2) encuestas dirigidas a los visitantes del área y (3) una tabla de puntaje aplicada por los autores, denominada "Ficha de Evaluación del Uso Turístico".

\section{Entrevistas}

Se diseñaron entrevistas personales dirigidas a personas involucradas directamente con la administración de ambos operadores turísticos con características similares, para conocer mejor los antecedentes de cada uno y poder entender el desarrollo del manejo de las operaciones turísticas a lo largo del tiempo hasta la actualidad, con énfasis en las medidas de conservación como manejo de agua, disposición de residuos, ahorro de energía y métodos de sensibilización al visitante en relación al tema 


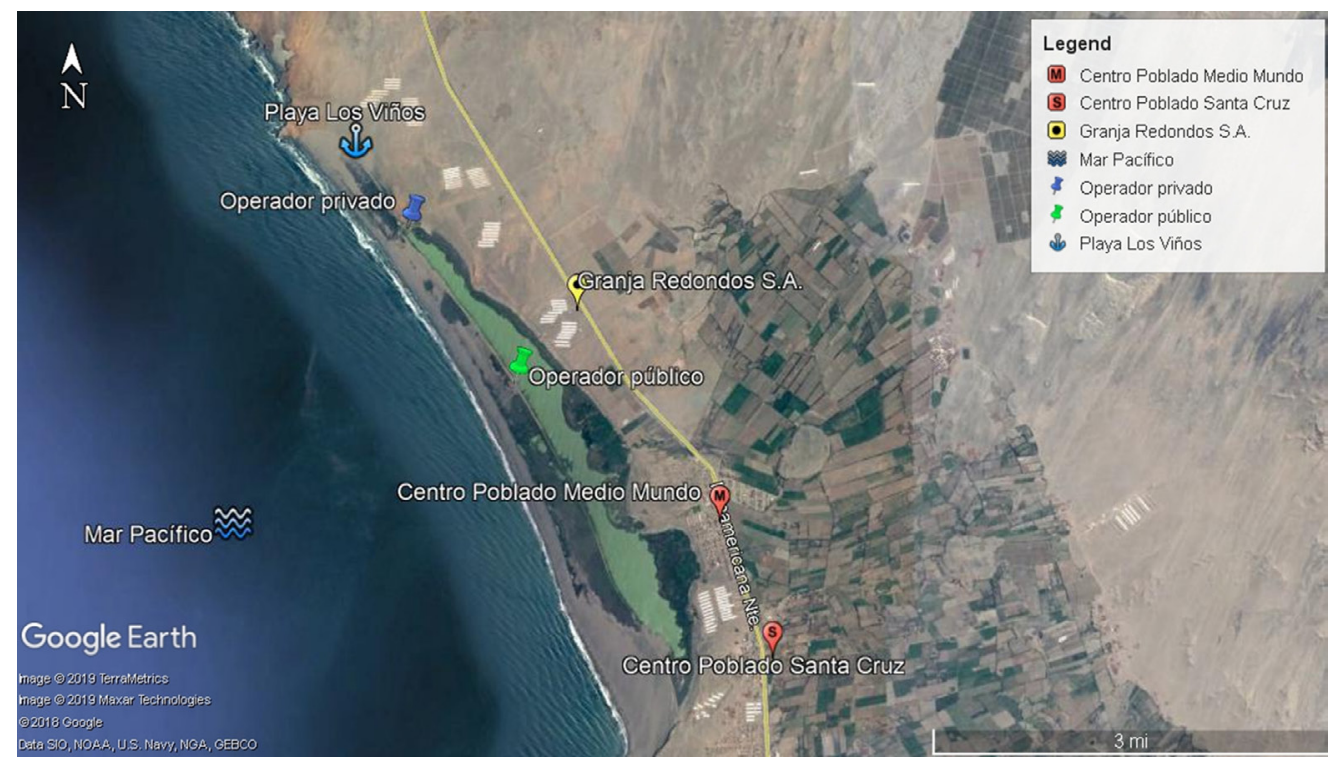

Figura 2. Ubicación de los operadores turísticos y límites del ACR Albufera de Medio Mundo.

de conservación, entre otros. Se entrevistaron a cuatro personas relacionadas a la administración y gestión del operador público y tres del operador privado. La información obtenida fue sistematizada y se mantuvo el anonimato de las respuestas. Las preguntas realizadas en las entrevistas personales son las siguientes:

1) ¿Cuáles son los cambios representativos del lugar en los últimos cinco años?

2) ¿Cuántos visitantes reciben al año? ¿Fechas de mayor acogida?

3) ¿Cuáles son los servicios que ofrecen y sus costos respectivos?

4) ¿Quién se encarga y cómo manejan el mantenimiento de sitio y las instalaciones?

5) ¿Cuáles son las medidas de conservación que aplican? os?

6) ¿Cómo manejan la disposición de residu-

7) ¿Apoyan las investigaciones que se realizan en el ACRAMM?

8) ¿Cuál es la relación con la población local?

9) ¿Contemplan un plan turístico?

\section{Encuestas}

Se adaptó la ficha de registro de visitantes y ficha de satisfacción del visitante de la Guía para la Gestión de Uso Público de los Atractivos del Circuito Turístico Nororiental (CTN PERÚ 2007) para diseñar una encuesta dirigida a los visitantes del ACRAMM (ver Anexo 1).

La muestra (n) fue de 43 visitantes que escogieron el operador turístico público y 43 visitantes que escogieron el operador privado. Este número fue determinado con la fórmula de poblaciones infinitas, ya que no se contaba con la cantidad de visitantes al área por año.

$$
n=\frac{Z^{2} \times p \times q}{E^{2}}
$$

\section{Donde:}

Z: Variable estandarizada $=1.96$ (usando un nivel de confianza del 95\%)

p: Varianza muestral positiva $=0.50$ (se asumen $50 \%$, al tener dos opciones de operadores turísticos)

q: Varianza muestral negativa $=0.50$ (se asumen $50 \%$, al tener dos opciones de operadores turísticos) 
Análisis comparativo de dos operadores turísticos en el

\section{E: Error estándar $=0.15$ (Análisis explorato-} rio)

Se realizaron un total de 86 encuestas durante los días 27, 28 y 29 de julio del 2018, al término del uso de los servicios turísticos, seleccionándose sólo visitantes mayores de edad (más de 18 años). Las primeras preguntas sin numeración fueron sobre información general, con la intención de obtener el perfil del visitante actual del ACRAMM: el operador elegido (público o privado), fecha, edad, sexo, nivel de instrucción y lugar de procedencia. En base a las preguntas número 1 y 2 se evaluó el grado de satisfacción de la visita y la opinión acerca de las facilidades en: (1) Entrada del sitio, (2) Señalización, (3) Información, (4) Servicio de guiado, (5) Mantenimiento del sitio, (6) Servicios higiénicos e (7) Infraestructura, con una calificación mínima de 1 punto que equivale a "Nada satisfecho" y máximo de 5 puntos que equivale a "Totalmente satisfecho". De esta forma, al otorgarle un puntaje a las respuestas cualitativas, la calificación de los criterios puede ser analizada cuantitativamente.

Los puntajes obtenidos se procesaron con el programa IBM SPSS Statistics 23, para calcular la media aritmética o promedio, la moda y la sumatoria para las respuestas de cada pregunta y se elaboró un cuadro resumen. De este modo, se comparó con medidas estadísticas la satisfacción y opinión de los visitantes sobre la calidad de las operaciones y servicios turísticos.

Finalmente, la pregunta número 3 fue una pregunta abierta en la que el visitante encuestado pudo hacer sugerencias de mejora a la administración del operador turístico elegido. Se elaboraron tablas de frecuencia con las recomendaciones que tuvieron al menos una repetición para cada operador. En cada encuesta, se consideró múltiples recomendaciones.

\section{Ficha de Evaluación del Uso Turístico}

Se realizó una visita de campo para establecer criterios de evaluación en función a las características específicas del área y los servicios que ambos operadores ofrecen a los visitantes, como: (1) Servicio de hospedaje, (2) Servicio de recorrido turístico y guiado, (3)
Servicio de información, (4) Servicio de alimentación, (5) Instalaciones y equipamientos, (6) Infraestructura, (7) Mantenimiento del sitio, (8) Aplicación de medidas de conservación, (9) Inclusión de la población local y (10) Planificación.

En base a estos criterios de evaluación se diseñó una tabla de puntaje denominada "Ficha de Evaluación del Uso Turístico" que contiene indicadores de calidad para cada criterio (ver Anexo 2). Estos diez criterios fueron calificados de manera ascendente, en función de la calidad, de la siguiente manera: 1 = "crítica" (color rojo), 2 = "deficiente" (color anaranjado), 3 = "aceptable" (color amarillo), 4 = "buena" (color verde claro) y 5 = "excelente" (color verde oscuro). Resultando en un puntaje total mínimo de 10 y máximo de 50 puntos. Además, se calificó la "Tendencia” de cada criterio evaluado en base a los "Enunciados estandarizados para describir las tendencias" de las Fichas de Evaluación Ecológica de Áreas Naturales Protegidas Del Noroeste de México (Comisión Nacional de Áreas Naturales Protegidas 2016) (ver Anexo 3).

Durante los días 27, 28 y 29 de julio del 2018, se aplicó la Ficha de Evaluación del Uso Turístico al operador público y privado para evaluar la calidad de las operaciones turísticas, en base a los diez criterios establecidos y justificando por escrito la selección de la calificación otorgada en función de los indicadores de calidad desarrollados para cada criterio. Además, se realizó un registro fotográfico de los indicadores más resaltantes para argumentar con hechos las calificaciones elegidas y mantener la objetividad de la evaluación.

Los resultados fueron procesados con el programa Excel 2016 y se elaboró una ficha de presentación a través de la página web CANVA (https://www.canva.com/) en base al modelo de las Fichas de Evaluación Ecológica de Áreas Naturales Protegidas del Noroeste de México (Comisión Nacional de Áreas Naturales Protegidas 2016) para poder analizar comparativamente la información e identificar los indicadores y variables que determinan un mayor $y$ 
menor puntaje en cada rubro, y así conocer los aspectos positivos y negativos de cada operador (ver Figura 4).

Finalmente, se realizó un análisis comparativo de los resultados procesados de las entrevistas, las encuestas y la "Ficha de Evaluación del Uso Turístico" para cada operador, junto con bibliografía revisada (UNWTO 2017, CTN PERÚ 2007, GORE Lima 2014, INC 2004, Severiche-Sierra et al. 2016, MINCETUR 2017, IUCN 2018, PromPerú y APTAE 2006, De la Torre 2011) para determinar cuál operador turístico ofrece un servicio de mejor calidad y con información suficiente para que el visitante comprenda la importancia del ACRAMM y el valor de la inversión realizada con su visita, de forma que se logre en él un cambio de actitud hacia un comportamiento favorable a la contribución de la conservación del ACR. Asimismo, se identificaron los criterios evaluados que obtuvieron menor y mayor calificación para cada operador turístico, y en base a estos, se elaboraron sugerencias de mejora y optimización con enfoque de conservación del área.

\section{Resultados}

\section{Entrevistas}

En cuanto a los resultados de las entrevistas, se resaltan los esfuerzos del Gobierno Regional de Lima por la concientización y sensibilización de la población local sobre la importancia del ACRAMM, como, por ejemplo, con el concurso de fotografía sobre la biodiversidad, que organiza como estrategia de puesta en valor del ACRAMM y también con la implementación de letreros informativos como parte de la concientización ambiental de los visitantes. No obstante, se comenta la falta de implementación de un Centro de Interpretación en el área.

En cuanto a las medidas de conservación que el operador público aplica, destacan las campañas de limpieza de la laguna que realiza con apoyo del GORE Lima, en las cuales invitan a la población local a participar. Asimismo, ha realizado la plantación de 100 plantones en el área del acantilado, ubicado al límite del ACR con el Centro Poblado de Medio Mundo para evitar que se arrojen desperdicios y desmonte en la zona. Sin embargo, se destaca la mayor acumulación de basura y escombros en el área que corresponde al operador público (obs. per.).

En cuanto al operador turístico privado, respecto a las medidas de concientización, cabe señalar la reciente implementación de un paseo turístico guiado en el que se utilizan paneles informativos para educar a los visitantes sobre la diversidad biológica en flora y fauna y los usos de las especies de flora, como el junco (Schoenoplectus americanus) y la totora balsa (Schoenoplectus californicus). Además, este operador ha desarrollado una "política de conservación" al aplicar medidas como el uso de energía eléctrica restringido a un horario (7 a $10 \mathrm{pm}$ ) para reducir el impacto sobre la avifauna, también ha implementado un programa de reciclaje de chapas de botellas de plástico para apoyar a la asociación "Angelito de Cristal" y las botellas de las mismas, son utilizadas como macetas para las plantas que producen en su vivero. Asimismo, cuenta con una pequeña hidroeléctrica, para aprovechar el desfogue de la laguna y utilizar "energía limpia". Además, han organizado un concurso escolar de dibujo como estrategia de puesta en valor del ACRAMM.

Por otro lado, la disposición de los residuos es un tema que requiere urgentemente atención para ambos operadores. Para el operador público, la administración de la $\mathrm{Mu}-$ nicipalidad de Végueta se encarga de dicha operación con mayor frecuencia durante el verano (temporada alta). Sin embargo, el operador privado menciona que prefiere almacenar los residuos de plástico, fomentar que los visitantes se lleven consigo sus residuos, quemar los papeles y hacer compost con los residuos orgánicos de la cocina, ya que la $\mathrm{Mu}$ nicipalidad de Végueta recoge los residuos y los acumula en un basural, en la entrada de Huacho, para incinerarlos.

Finalmente, ambos operadores turísticos carecen de una planificación turística eficiente. Aunque el GORE Lima desarrolló el Plan Maestro 2015-2019 del ACRAMM, que 
Análisis comparativo de dos operadores turísticos en el

contempla la construcción de un Centro de Interpretación, la implementación de puestos de control y la renovación de los hitos, dichas actividades aún no se han ejecutado. Por otro lado, el operador privado realiza "administración tipo hotelera": manejan un libro de reclamaciones, practican la "cadena de frío" para los pescados y mariscos y mantienen buenos estándares de salubridad para los insumos.

\section{Encuestas}

El visitante más común, que escoge tanto el operador público como el operador privado, para visitar el ACRAMM es un adulto (entre 34 y 60 años de edad), mujer, proveniente del departamento de Lima y que cursa un nivel de instrucción superior (técnico/universitario) al momento de su visita. De lo observado en campo, los grupos que escogen el operador público durante Fiestas Patrias, visitan el ACR como parte de un paquete turístico tipo " $f u l l$ day" ofrecido por diferentes agencias de turismo, que incluye la Reserva Nacional de Lachay y la Zona Arqueológica Caral, por lo que no pernoctan en las instalaciones que ofrece el operador público. En cambio, los visitantes que escogen el operador privado casi en su totalidad pernoctan.

Al comparar los resultados de ambos operadores (ver Cuadro 1), se observa que las medidas estadísticas; media, moda y sumatoria, tanto de satisfacción general de la visita como de opinión sobre las siete facilidades evaluadas, son mayores para el operador privado que para el público.

En cuanto a la satisfacción de la visita, la calificación promedio otorgada por los visitantes encuestados para el operador turístico público fue 3,79, mientras que para el operador turístico privado fue 4,49 . Es decir que la calificación promedio de la satisfacción de la visita fue mayor para el operador privado. Además, el puntaje otorgado con mayor frecuencia para el operador público fue 4,00 y para el privado fue 5,00; lo que quiere decir que el puntaje que se repitió más entre los visitantes encuestados del operador privado fue la máxima calificación. El puntaje total para el operador privado fue

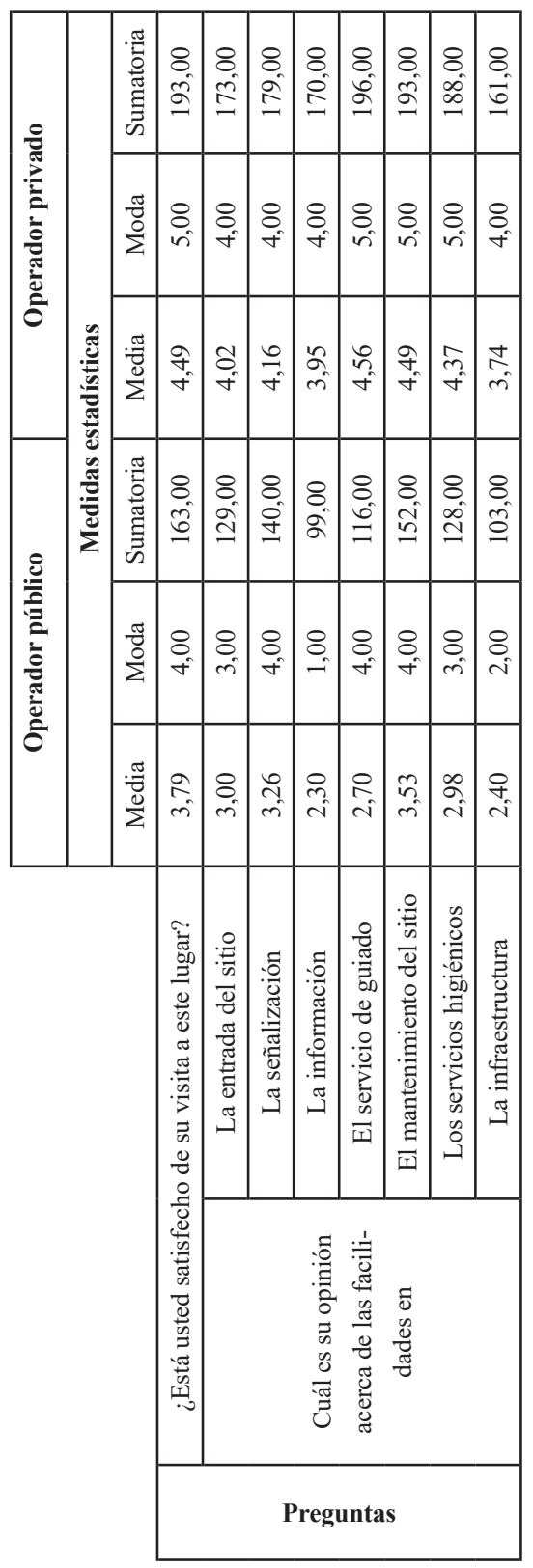

Cuadro 1. Medidas estadísticas de los resultados de satisfacción y opinión acerca de las facilidades del ACRAMM para los visitantes que escogieron el operador público y privado. 


\begin{tabular}{|c|c|c|c|}
\hline $\begin{array}{l}\text { Grupo de recomendación para } \\
\text { el operador público }\end{array}$ & $\begin{array}{l}\text { Porcentaje } \\
\quad(\%)\end{array}$ & $\begin{array}{l}\text { Grupo de recomendación para } \\
\text { el operador privado }\end{array}$ & $\begin{array}{l}\text { Porcentaje } \\
\quad(\%)\end{array}$ \\
\hline Mantenimiento del sitio & 14,30 & Señalización de la pista de acceso & 23,10 \\
\hline Información & 10,70 & Publicidad & 17,90 \\
\hline Señalización & 8,30 & Señalización & 12,80 \\
\hline Servicio de alimentación & 7,10 & Luz permanente & 10,30 \\
\hline Servicios higiénicos & 7,10 & Información & 10,30 \\
\hline Servicio de guiado & 7,10 & Mantenimiento del sitio & 7,70 \\
\hline Señalización de la pista de acceso & 6,00 & $\begin{array}{c}\text { Mayor diversidad de actividades } \\
\text { en el sitio }\end{array}$ & 7,70 \\
\hline Organización y orden & 6,00 & $\begin{array}{l}\text { Variedad en el servicio de ali- } \\
\text { mentación }\end{array}$ & 5,10 \\
\hline Infraestructura & 6,00 & Servicios higiénicos & 2,60 \\
\hline Seguridad & 6,00 & Infraestructura & 2,60 \\
\hline Personal capacitado & 4,80 & Total & 100,00 \\
\hline Imagen del sitio & 3,60 & & \\
\hline Publicidad & 3,60 & & \\
\hline Entrada del sitio & 2,40 & & \\
\hline Atención al visitante & 2,40 & & \\
\hline Adaptación para niños & 2,40 & & \\
\hline $\begin{array}{l}\text { Mayor diversidad de actividades } \\
\text { en el sitio }\end{array}$ & 2,40 & & \\
\hline Total & 100,00 & & \\
\hline
\end{tabular}

Cuadro 2. Recomendaciones por grupos de los visitantes al ACRAMM que escogieron el operador público y privado.

163,00 y para el privado 193,00 , lo que representa 30 puntos más para el operador privado.

Respecto a la opinión acerca de las facilidades brindadas por el operador público, los resultados de las medidas estadísticas con mayores puntajes corresponden al criterio mantenimiento del sitio, cuya calificación promedio fue 3,53; el puntaje otorgado con mayor frecuencia por los encuestados fue 4,00; y la calificación total fue 152,00 puntos. Los resultados con menores puntajes corresponden al criterio información, cuya calificación promedio fue 2,30; el puntaje otorgado con mayor frecuencia por los encuestados fue $1,00 \mathrm{y}$ la calificación total fue 99,00 puntos.
Los resultados de las medidas estadísticas con mayores puntajes para el operador privado corresponden al criterio servicio de guiado, cuya calificación promedio fue 4,56; el puntaje otorgado con mayor frecuencia por los encuestados fue 5,00 y la calificación total fue 196,00 puntos. Los resultados con menores puntajes corresponden al criterio infraestructura, cuya calificación promedio fue 3,74 ; el puntaje otorgado con mayor frecuencia por los encuestados fue 4,00 y la calificación total fue 161,00 puntos.

Respecto a las recomendaciones que realizaron los encuestados (ver Cuadro 2), se puede observar notoriamente, que existen más grupos 
Análisis comparativo de dos operadores turísticos en el

de recomendaciones para el operador público que para el operador privado. A pesar que el criterio mejor calificado en las encuestas para el operador público fue el mantenimiento del sitio, contradictoriamente, la recomendación de mayor frecuencia fue mejorar el "Mantenimiento del sitio". Por otro lado, las recomendaciones más frecuentes para el operador privado fueron mejorar la "Señalización de la pista de acceso" y la "Publicidad".

\section{Ficha de Evaluación del Uso Turístico}

Para el operador público el criterio que recibió mayor calificación fue la "aceptable" inclusión de la población local, con tendencia de "en mejoría" ya que los empleados en su totalidad provienen del Centro Poblado de Medio Mundo. Asimismo, los restaurantes son concesionarios locales y el servicio de paseo en bote es manejado en parte por la Asociación de Pescadores. Sin embargo, la organización es bastante informal y tiene mucho mayor potencial de inclusión, por ejemplo, se podría realizar la venta del arte que produce la "Asociación de Mujeres Artesanas de Medio Mundo" $u$ otras organizaciones de artistas en un centro de venta en las instalaciones del operador público.

Mientras que, los criterios de menor calificación fueron el "crítico" servicio de información, con tendencia "en mejoría" debido a que no existe material visual didáctico que sirva como herramienta para que el visitante conozca sobre la importancia del área y su conservación. Tampoco se entrega material escrito, como mapas o trípticos informativos a los visitantes. Además, hace falta un espacio que sirva como Centro de Interpretación propiamente dicho.

La "crítica" planificación, con tendencia "estable", ya que la administración del sitio carece de un plan de uso turístico que incluya concientización de visitante, sistema de registro de visitantes o sistema de monitoreo de consumo de agua o luz. El personal no recibe capacitación. No cuentan con un sistema de reporte de daños de las instalaciones y equipos, ni manejan un libro de reclamaciones o buzón de sugerencia. El sitio carece de un plan de evacuación en caso de emergencia y falta personal de seguridad. En general denota bastante ausencia de organización, probablemente a causa de la poca presencia del GORE Lima. Cabe mencionar que el ACR, debido a sus características, tiene potencial para ser sede de eventos deportivos de remo, pero carece de la organización adecuada para dicho uso.

Por otro lado, para el operador privado, el criterio que recibió mayor calificación fue el "superior" servicio de alimentación, con tendencia "estable" porque la cocina se encuentra limpia y la administración le da mucha importancia a la salubridad, por ejemplo, con el método de la "cadena fría" que utilizan para mantener frescos los mariscos y pescados. El restaurante tiene un ambiente agradable. El sabor de la comida es muy bueno y los precios son proporcionales a la calidad de servicio.

Mientras que, los criterios de menor calificación fueron las "aceptables" instalaciones y equipamiento, con tendencia de "en mejoría" porque la señalización es apropiada, ya que los carteles están hechos con materiales acorde al lugar, llevan mensajes claros y sencillos y se ven cuidados. Sin embargo, otros equipos como los contenedores de basura no se ven en sintonía con el ambiente. Podrían estar hechos de materiales locales, en lugar de plástico. Por otro lado, los baños comunes y privados están bien equipados con papel higiénico, papel toalla o jabón. Asimismo, el estacionamiento está señalado correctamente.

La "aceptable" inclusión de la población local, con tendencia "estable" ya que los empleados provienen del Centro Poblado de Medio Mundo y las ciudades de Barranca y Huacho. Además, con el Programa Vivencial de Ciencias Albuferas de Medio Mundo (PROVICAMP) promueven que los estudiantes, tanto escolares como universitarios locales, conozcan el ACRAMM y la importancia de su conservación. Sin embargo, la relación con la población local podría mejorar, por ejemplo, con la promoción de compra de productos de arte local en el centro de ventas del complejo hotelero (ver Figura 3). 


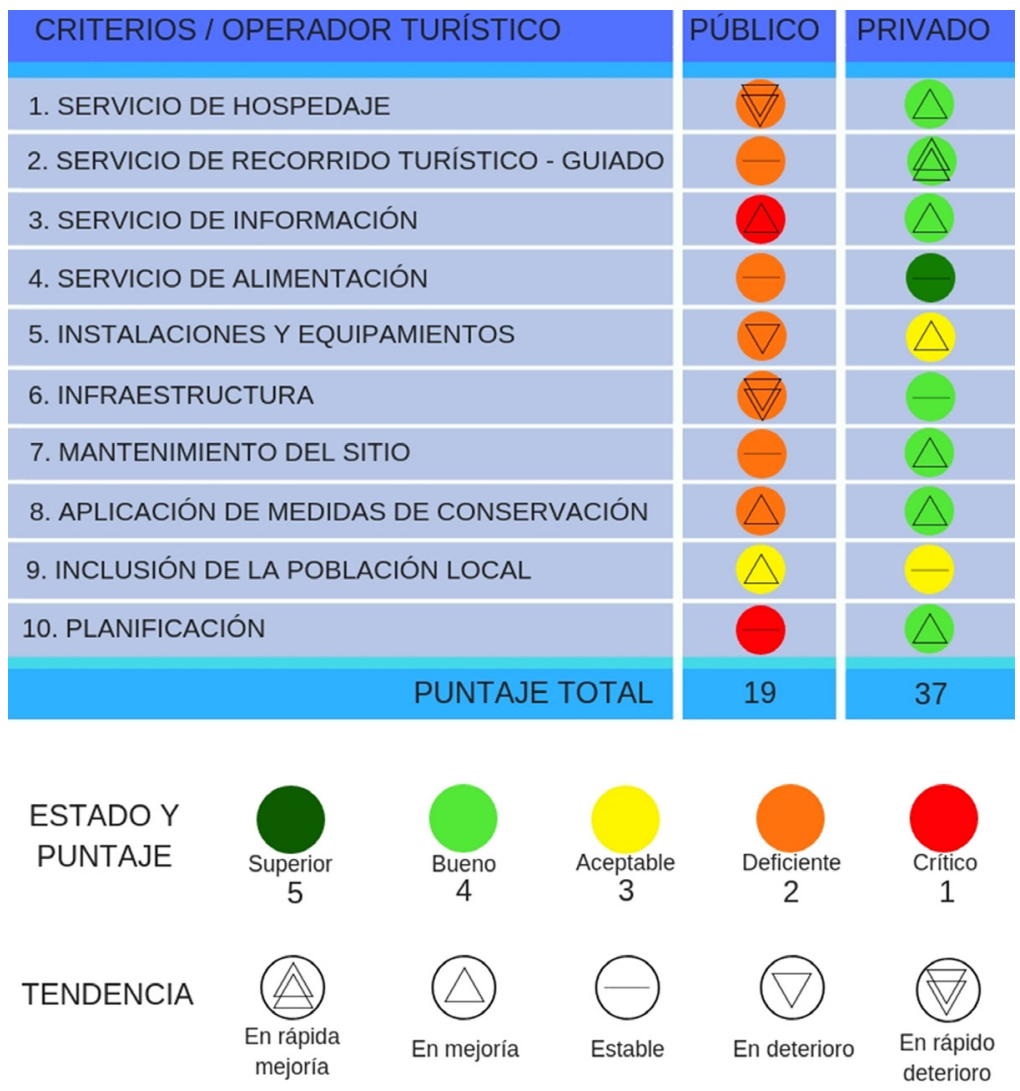

Figura 3. Ficha de Evaluación del Uso Turístico del Operador público y privado del ACRAMM.

\section{Discusión}

\section{Operador público}

A partir de las entrevistas, encuestas y ficha de evaluación del uso turístico, se puede evidenciar que el operador turístico público desarrolla un deficiente uso turístico del ACRAMM porque depende en gran medida del GORE Lima y carece de planificación turística, reflejándose en la ausencia de organización y los bajos estándares de calidad de servicios que ofrece. Tomando en cuenta que, la calidad se mide desde la apreciación del turista, es decir, todo se resume en la satisfacción y superación de las necesidades y requerimientos que tenga el turista (Castilla y Alarcón 2017), este ope- rador no satisface las necesidades, exigencias y expectativas legítimas de los consumidores ni se desarrolla una actividad turística preocupada por su entorno humano y natural (UNWTO 2017).

Los siguientes aspectos han sido identificados como más resaltantes: (1) la falta de un Centro de Interpretación, lo que dificulta la transmisión de mensajes sobre la conservación del sitio, a pesar de los esfuerzos de concientización y sensibilización que desarrolla el GORE Lima, como el concurso escolar de fotografía y las campañas de limpieza del área con la población local. También, (2) el mantenimiento del sitio, que resultó el criterio con mayor calificación en las encuestas (calificación promedio de 3,53), 
Análisis comparativo de dos operadores turísticos en el

pero que contradictoriamente fue la recomendación más frecuente entre los encuestados y (3) la inclusión de la población local, que resultó el criterio mejor calificado en la ficha de evaluación del uso turístico (calificado como "aceptable" y con tendencia "en mejoría"), debido a la buena relación que mantiene este operador con la población local.

Sin embargo, los resultados de esta investigación evidencian también que el operador público no desarrolla planificación turística eficiente porque no ejecuta acciones y actividades de manera ordenada, con una visión a futuro, tanto en el corto, mediano y largo plazo, ni orienta su administración hacia la regulación de la actividad turística y recreativa, según las prioridades de conservación y manejo del área (CTN PERÚ 2007). Debido a que, a pesar de contar con un Plan Maestro, este no propone lineamientos específicos para la ejecución de la actividad turística. Este hecho se ve reflejado en las deficiencias, por ejemplo, del servicio de información, que resultó como el criterio con menor calificación en las encuestas (calificación promedio de 2,30) y fue calificado como "crítico", con tendencia de "en mejoría" en la ficha de evaluación del uso turístico debido a que este operador no utiliza herramientas eficientes de comunicación para llegar al visitante: no existe infografía, material visual o escrito con información pertinente sobre el área y que sirva de apoyo a la explicación del guía. Por lo tanto, el criterio de planificación fue calificado como "crítico" en la ficha de evaluación del uso turístico porque, a pesar de ser un ACR, carece de un plan de uso turístico que establezca las estrategias para lograr objetivos definidos, como: la concientización y educación ambiental al visitante, constante capacitación de los trabajadores, mantenimiento de las instalaciones y equipos, así como mecanismos de puesta en valor de los recursos, métodos de monitoreo de consumo de agua o luz, manejo de libro de reclamaciones o buzón de sugerencia, plan de evacuación en casos de emergencia, seguridad, entre otros.

Cabe mencionar que, las deficiencias mencionadas, denotan que este operador no prac- tica la "puesta en valor", que es una forma de revalorizar un bien o recurso cultural para que la sociedad aprecie su valor y así poder protegerlo, a través de acciones como recuperación, interpretación y difusión de información, con el objetivo de mantener el recurso disponible para el disfrute del visitante (INC 2004). De esta forma, el visitante entiende que, con el pago de una entrada, la contratación de servicios, el uso de la infraestructura, entre otros, contribuye con la preservación del patrimonio visitado y así se genera en el visitante un sentimiento de contribución a un fin mayor, que es la conservación del sitio.

\section{Operador privado}

Por otro lado, a partir de las entrevistas, encuestas y ficha de evaluación del uso turístico, se puede evidenciar que el operador turístico privado desarrolla un eficiente uso turístico del ACRAMM porque ejerce administración hotelera, lo cual se refleja en los altos estándares de calidad de servicios que ofrece debido a que satisfacen las necesidad, exigencias y expectativas legítimas de los consumidores y se desarrolla una actividad turística preocupada por su entorno humano y natural (UNWTO 2017).

Los siguientes aspectos han sido identificados como más resaltantes: (1) las medidas de conservación, ya que aplica diversas estrategias de concientización para los visitantes y la población local, como el uso de energía eléctrica restringida, el reciclaje de chapas y botellas de plástico para apoyar a la asociación "Angelito de Cristal" y la producción de plantas en el vivero, el uso de energías limpias a través de la pequeña hidroeléctrica y el concurso escolar de dibujo para la puesta en valor del atractivo turístico visitado. También, (2) el servicio de recorrido turístico y guiado, que resultó el criterio con mayor calificación en las encuestas (calificación promedio de 4,56), debido a que utilizan materiales de apoyo como paneles informativos para educar a los visitantes sobre la diversidad de recursos del área. Y (3) el servicio de alimentación, que resultó el criterio mejor calificado en la ficha de evaluación del uso turístico (calificado como "superior" y con 
tendencia "estable"), como consecuencia de la limpieza, salubridad y utilización de los residuos orgánicos de la cocina para la producción de compost.

Las prácticas mencionadas, forman parte del concepto de educación ambiental, considerado como pilar fundamental de la generación de cambio de actitud y aptitud y de lograr el equilibrio entre el ser humano y su entorno (Severiche-Sierra et al. 2016). Asimismo, cabe mencionar que este tipo de educación puede entenderse como "educación para la conservación" pues trata de integrar un conjunto de estrategias dirigidas a la formación de personas comprometidas con su propio desarrollo individual y colectivo a partir del crecimiento, respeto y relación con su medio ambiente (MINCETUR 2017), por lo tanto, constituye una herramienta imprescindible para transmitir de manera eficiente mensajes a los visitantes sobre la importancia y necesidad de preservar el ACRAMM. Es decir que, el operador privado logra a través de la calidad superior de las medidas de conservación aplicadas y los servicios de recorrido turístico y alimentación ofrecidos, un cambio de actitud de los usuarios hacia el desarrollo de mayor conciencia y respeto por la naturaleza.

Sin embargo, los resultados de esta investigación evidencian también que el operador privado aún no desarrolla en su totalidad turismo sostenible debido a que no responde completamente a las necesidades del ambiente y las comunidades locales (IUCN 2018), porque presenta deficiencias en la disposición de residuos, ya que almacenan los residuos de plástico, las instalaciones y equipamiento que ofrecen a los visitantes no son totalmente acorde al sitio y sobre todo, no practican eficientemente la inclusión de la población local, por ejemplo con la promoción de la venta del arte local. Cabe mencionar que, a través de los resultados de las encuestas, los visitantes recomiendan mejorar la señalización de acceso a las instalaciones y la publicidad.

Bajo el concepto de que la calidad en el turismo es el resultado de un proceso que im- plica la satisfacción de todas las necesidades, exigencias y expectativas legítimas de los consumidores respecto a los productos y servicios $\mathrm{y}$ con los factores que determinan la calidad, tales como la seguridad, la higiene, la accesibilidad, la transparencia, la autenticidad y la armonía de una actividad turística preocupada por su entorno humano y natural (UNWTO 2017), los resultados obtenidos de las entrevistas, encuestas y fichas de evaluación indican que las operaciones y servicios turísticos que ofrece el operador privado son de mayor calidad y satisfacen más a los visitantes ya que las calificaciones de satisfacción de visita, opinión de las facilidades y puntajes de la Ficha de Evaluación del Uso Turístico fueron en general mayores para este. Por lo tanto, el operador privado ofrece servicios turísticos de mayor calidad que el operador público, sobre todo el servicio de recorrido turístico guiado, en el cual utilizan mecanismos de concientización de los visitantes como la difusión de información sobre las características del sitio que lo convierten en un área natural protegida, desarrollando así una actividad turística preocupada por su entorno humano y natural. Es decir que, utilizan la sensibilización para crear conciencia y lograr la motivación de los trabajadores y los visitantes, como parte de la "buenas prácticas en el turismo" (PromPerú y APTAE 2006).

Finalmente, cabe mencionar que la calidad en los servicios turísticos implica revisión de los procesos, cambio de filosofía, nueva cultura organizacional, inversión en el proceso de mejora, entrenamiento y el convencimiento de que la satisfacción del turista debe ser la prioridad (De la Torre 2011). Es decir que, la metodología usada en esta investigación puede servir como un ejemplo para evaluar la calidad de los servicios y operaciones turísticas, por medio de entrevistas, encuestas y fichas de evaluación del uso turístico elaboradas en función de las características específicas de sitio. De esta forma, es posible evaluar estándares de calidad en el desarrollo del ecoturismo en las áreas naturales protegidas y áreas silvestres, desde un enfoque cuantitativo. 
Análisis comparativo de dos operadores turísticos en el

\section{Conclusiones}

Las operaciones turísticas que ejecuta el operador privado son de mayor calidad que las del operador público, lo que le permite desarrollar un uso turístico del área más eficiente, en pro de la conservación del Área de Conservación Regional Albufera de Medio Mundo. Es decir que, los visitantes que escogen el operador privado tienen una experiencia de visita más satisfactoria, sobre todo porque en el servicio de recorrido turístico y guiado se prioriza la trasmisión de mensajes sobre la importancia de la conservación del sitio. De esta manera, es posible que los visitantes comprendan los valores del ACR e incentivar que desarrollen una actitud favorable a la necesidad de conservarlo. Sin embargo, el operador privado debe mejorar sus prácticas en disposición de residuos e inclusión de la población local para lograr un turismo sostenible. Mientras que, el operador público debe mejorar su planificación turística para mejorar en general los procesos de los servicios turísticos que ofrecen, estandarizarlos y monitorearlos y así, mantener estándares de calidad más altos.

\section{Agradecimientos}

Los autores agradecen a al Gobierno Regional de Lima, a la Municipalidad Distrital de Végueta y a la familia Rovegno, del complejo hotelero "Albuferas de Medio Mundo Bungalows \& Camping", por su apoyo durante la realización de este estudio. Asimismo, a los ingenieros Pedro Vásquez y Antonio Tovar por el apoyo incondicional y la buena disposición.

\section{Bibliografía}

Aníbal, P y Baez, S. 2018. Medición de la experiencia del turista en Quito. Revista Brasileira de Pesquisa em Turismo. Sao Paulo, BR. 12(1):133-156.

Aponte, H. 2017. Humedales de la Costa Central del Perú. Un diagnóstico de los humedales Santa Rosa, laguna El Paraíso y Albufera de Medio Mundo. Lima, PE. CooperAcción. 116 p.
Aponte, H y Cano, A. 2018. Flora Vascular del Humedal del Carquín - Hualmay, Huaura (Lima, Perú) (en línea). Revista Ecología Aplicada 17(1):69-76. Consultado el 09 set. 2019. Disponible en http://www.lamolina.edu.pe/ ecolapl/08 Articulo Vol 17 no1.pdf

Aponte, H; Jiménez R. y Alcántara B. 2012. Challenges for management and conservation of Santa Rosa Wetland. Científica 9(3):257264.

Castilla, G. y Alarcón N. 2017. Evaluación de la Calidad en Establecimientos de Alojamiento y Hospedaje (en línea). Revista Semestre Económico 20(43):161-190. Consultado el 09 set. 2019. Disponible en http://www. scielo.org.co/pdf/seec/v20n43/0120-6346seec-20-43-00161.pdf

Comisión Nacional de Áreas Naturales Protegidas. 2016. Fichas de Evaluación Ecológica de Áreas Naturales Protegidas Del Noroeste de México. MX. 240 p. (en línea). Consultado 12 set. 2018. Disponible en https://simec.conanp. gob.mx/pdf score/1.pdf

CTN PERÚ (Circuito Turístico Nororiental). 2007. Guía para la Gestión de Uso Público de los Atractivos del Circuito Turístico Nororiental. Cajamarca, PE. Publisher SRL. 44 p.

Decreto supremo N 006-2007-AG. 2007. Establecen el Área de Conservación Regional "Albufera de Medio Mundo", ubicada en la provincia de Huaura, departamento de Lima. Diario Oficial El Peruano. Perú. 25 ene.

De la Torre, A. 2011. Turismo y Calidad del Servicio. Escuela Profesional de Turismo y Hotelería. Revista Cultura 25:127-142.

GORE Lima (Gobierno Regional de Lima). Gerencia Regional de Recursos Naturales y Gestión del Medio Ambiente. 2009. Plan Maestro del Área de Conservación Regional Albufera de Medio Mundo 2009-2013 (en línea). Consultado 09 may. 2018. Disponible en http:// www.regionlima.gob.pe/imatemporales/Plan Maestro Albufera de Medio Mundo.pdf

GORE Lima (Gobierno Regional de Lima). Gerencia Regional de Recursos Naturales 
y Gestión del Medio Ambiente. 2014. Plan Maestro del Área de Conservación Regional Albufera de Medio Mundo 2015-2019 (en línea). Consultado 09 may. 2018. Disponible en http://siar.regionlima.gob.pe/sites/default/ files/archivos/public/docs/plan-maestro-acramm-1-30.pdf

Hernández, F; Vargas, J y Aguilar, J. 2015. El efecto emocional de una experiencia turística: el mercado de artesanías de Oaxaca, México. Cathedra et Scientia. International Journal 1(1):147-154.

IUCN (International Union for Conservation of Nature and Natural Resources). 2018. Tourism and Visitor Management in Protected Areas: Guidelines for Sustainability. Best Practice Protected Area Guidelines. L Yu-Fai. A Spenceley. G Hvenegaard y R Buckley. Gland, CH. IUCN. 128 p.

INC (Instituto Nacional de Cultura). 2004. El lugar sin límites. Gaceta cultural del Perú 8:3.

MINAGRI (Ministerio de Agricultura y Riego). 2007. Establecen el Área de Conservación Regional "Albufera de Medio Mundo" ubicada en la provincia de Huaura, departamento de Lima. Decreto Supremo Na006-2007-AG. Consultado 21 may. 2018. Disponible en http://minagri.gob.pe/portal//download/pdf/ marcolegal/normaslegales/decretossupremos/ DECRETO\%20SUPREMO\%20N\%200062007-AG.pdf

MINAM (Ministerio del Ambiente). 2015. Estrategia Nacional de Humedales. Viceministerio de Desarrollo Estratégico de los Recursos Naturales. Lima, PE. Dirección General de Diversidad Biológica. $59 \mathrm{p}$.

MINCETUR (Ministerio de Comercio y Turismo) 2012. Manual de las Buenas Prácticas de Gestión de Servicios para Establecimiento de Hospedaje. Plan Nacional de Calidad Turística del Perú - CALTUR. Lima, PE. 76 p.

MINCETUR (Ministerio de Comercio Exterior y Turismo). 2017. Plan Nacional de Calidad Turística del Perú - CALTUR 2017-2025. Lima, PE. 34 p.
Moschella, P. 2012. Variación y Protección de Humedales Costeros Frente a Procesos de Urbanización: Casos Ventanilla y Puerto Viejo. Tesis M. Sc. Lima, PE. Pontificia Universidad Católica del Perú. 132 p. (en línea). Consultado 14 may. 2018. Disponible en http:// tesis.pucp.edu.pe/repositorio/bitstream/handle/20.500.12404/4527/MOSCHELLA MILOSLAVICH PAOLA VARIACION.pdf?sequence $=1$ \&isAllowed $=y$

Pacífico Adventures. 2016. Ballenas en el norte del Perú. Whales of Northern Peru. Lima, PE. Universidad Científica del Sur. 130 p.

PromPerú (Comisión de Promoción del Perú); APTAE (Asociación Peruana de Turismo de Aventura y Ecoturism). 2006. Manual de Buenas Prácticas para Turismo Sostenible. Hacia un Turismo Responsable. Lima, PE. 100 p.

ProNaturaleza. 2010. Documento Base para la Elaboración de una Estrategia de Conservación Humedales de la Costa Peruana. Lima (en línea). Consultado 11 may. 2018. Disponible en http://www.agrolalibertad.gob.pe/sites/default/ files/humedales de la costa peruanavf.pdf

Rivera, M. 2013. El turismo experiencial como forma de turismo responsable e intercultural. Relacionales interculturales en la diversidad. Universidad de Córdoba. Córdoba, ES. 199-217 pp.

Severiche-Sierra; Gómez-Bustamante; Jaimes-Morales. 2016. La educación como base cultural y estrategia para el desarrollo sostenible. Revista de Estudios Interdisciplinarios de Ciencias Sociales 18(2):266-281.

Sosa, L; Silvestre, M. 2018. Evaluación de la calidad de los servicios turísticos gastronómicos en los establecimientos de alimentos y bebidas de comida tradicional regional Colimota en Manzanillo, Colima (en línea). Revista El Periplo Sustentable 35:151-179. Consultado el 09 set. 2019. Disponible en http://www.scielo.org. mx/pdf/eps/n35/1870-9036-eps-35-151.pdf

UNWTO (World Tourism Organization). 2017. Practical Guidelines for Integrated Quality Management in Tourism Destinations Concepts, Implementation and Tools for Destination Management Organizations. 


\section{Anexos}

Anexo 1. Formato de encuesta a visitantes del ACR Albufera de Medio Mundo.

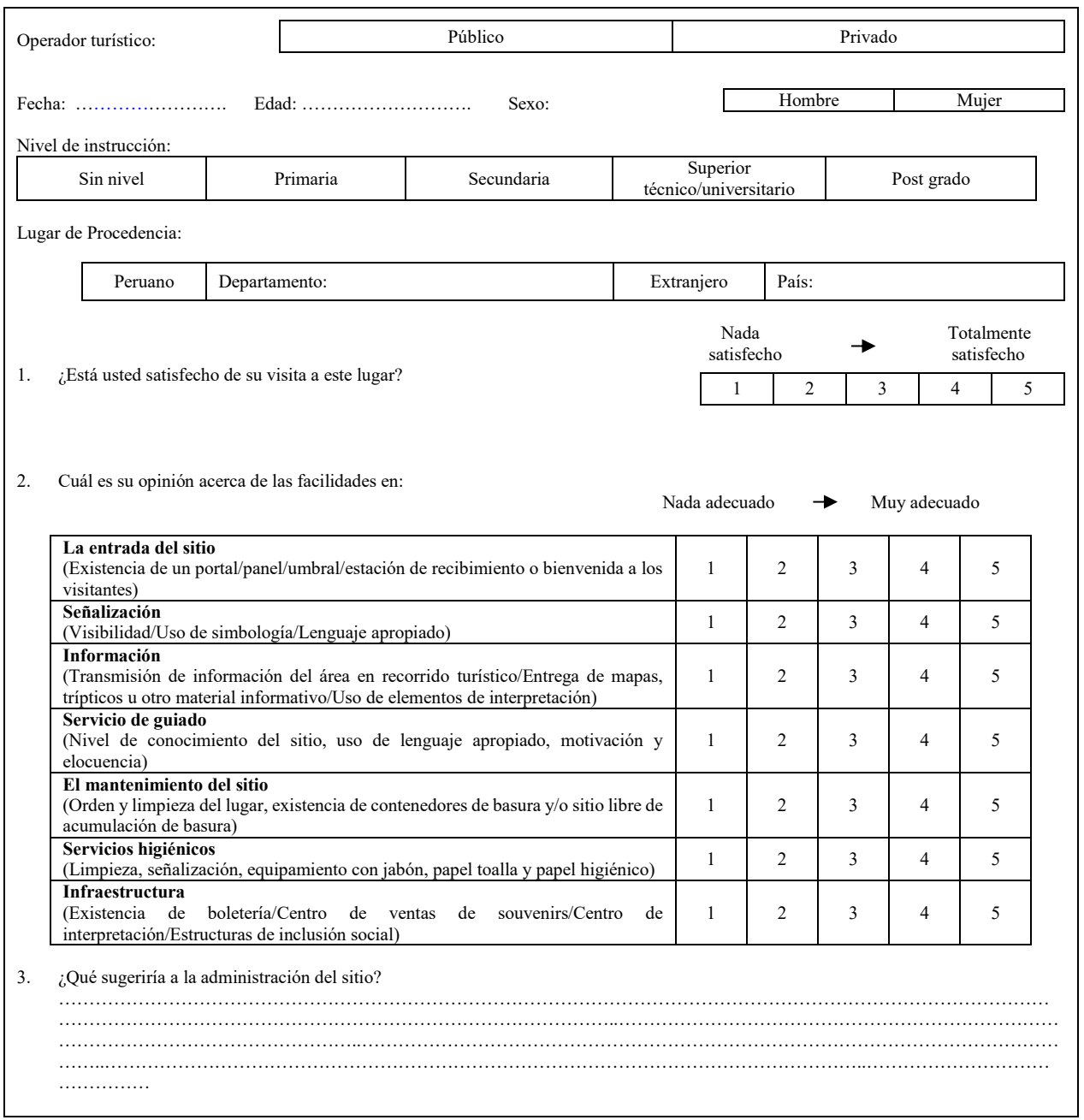


Anexo 2. Ficha de Evaluación del Uso Turístico.

\begin{tabular}{|c|c|c|c|c|c|c|c|c|c|c|}
\hline 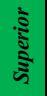 & in & in & in & in & in & in & in & in & in & in \\
\hline 气ิ๊ & $\nabla$ & t & $\nabla$ & $\sigma$ & $\nabla$ & $\nabla$ & $\nabla$ & 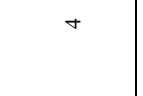 & $\sigma$ & t \\
\hline हैँ & $m$ & $m$ & $m$ & m & $m$ & m & $m$ & $m$ & $m$ & $m$ \\
\hline క్ & $\sim$ & $N$ & $\sim$ & $N$ & $N$ & $N$ & $\sim$ & $N$ & $N$ & $N$ \\
\hline & - & - & - & - & - & - & - & - & - & - \\
\hline 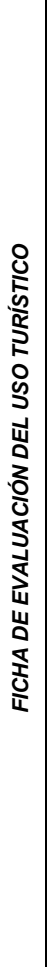 & 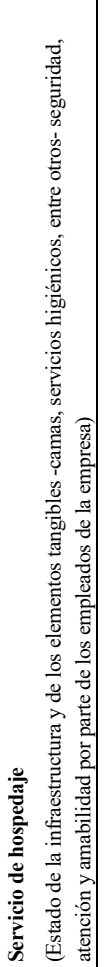 & 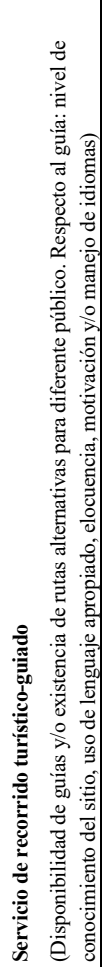 & 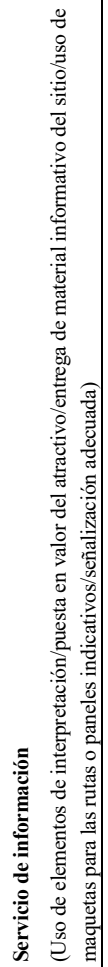 & 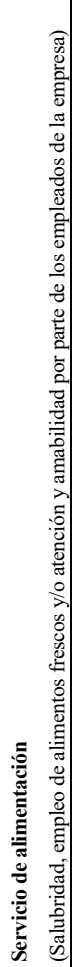 & 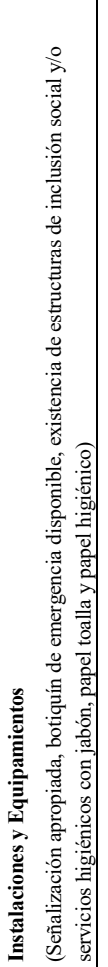 & 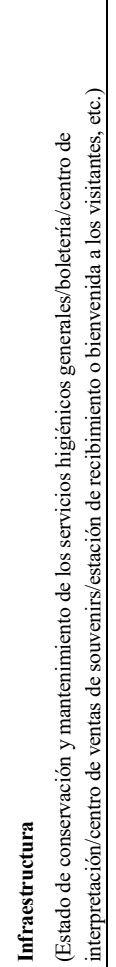 & 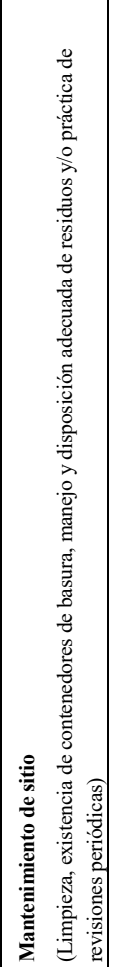 & 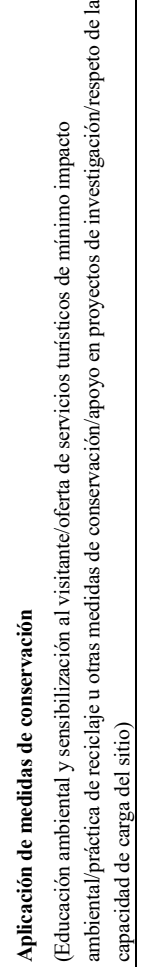 & 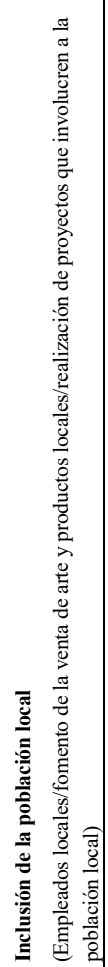 & 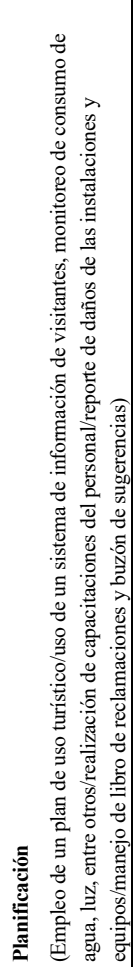 \\
\hline
\end{tabular}


Anexo 3. Enunciados estandarizados para describir las tendencias.

\begin{tabular}{|c|c|}
\hline Enunciado sobre la tendencia & Calificación \\
\hline $\begin{array}{c}\text { Aparentemente las condiciones están cambiando a un ritmo que } \\
\text { conducirá a un mejor estado en cinco años. }\end{array}$ & En rápida mejoría \\
\hline Las condiciones están mejorando & En mejoría \\
\hline $\begin{array}{c}\text { Dentro de los límites de la variación normal, no se prevén cambios } \\
\text { sistemáticos debido a fuentes antropogénicas o de otra índole. }\end{array}$ & Estable \\
\hline Las condiciones están empeorando. & En deterioro \\
\hline $\begin{array}{c}\text { Aparentemente las condiciones están cambiando a un ritmo que } \\
\text { conducirá a un deterioro del estado actual en cinco años. }\end{array}$ & En rápido deterioro \\
\hline
\end{tabular}

